\title{
Pantothenate auxotrophy of Methylobacterium spp. isolated from living plants
}

$\operatorname{AUTHOR}(\mathrm{S})$ :

Yoshida, Yusuke; Iguchi, Hiroyuki; Sakai, Yasuyoshi; Yurimoto, Hiroya

\section{CITATION:}

Yoshida, Yusuke ...[et al]. Pantothenate auxotrophy of Methylobacterium spp. isolated from living plants. Bioscience, biotechnology, and biochemistry 2018: 1549935.

\section{ISSUE DATE:}

2018-11-26

URL:

http://hdl.handle.net/2433/235623

\section{RIGHT:}

This is an Accepted Manuscript of an article published by Taylor \& Francis in Bioscience, biotechnology, and biochemistry on 26 November 2018, available online: http:/ www.tandfonline.com/10.1080/09168451.2018.1549935; The full-text file will be made open to the public on 26 November 2019 in accordance with publisher's 'Terms and Conditions for Self-Archiving', この論文は出版社版でありません。引用の際には出版社版をご確認ご利用ください。 This is not the published version. Please cite only the published version. 
1 Running title: Pantothenate auxotrophy of PPFM isolated from living plants

2

3

4

5

6

7

8 Yusuke Yoshida ${ }^{1}$, Hiroyuki Iguchi ${ }^{1,2}$, Yasuyoshi Sakai ${ }^{1}$, and Hiroya Yurimoto $^{1}{ }^{*}$

9

$10{ }^{1}$ Division of Applied Life Sciences, Graduate School of Agriculture, Kyoto University,

11 Kitashirakawa-Oiwake, Sakyo-ku, Kyoto 606-8502, Japan;

$12{ }^{2}$ Department of Agriculture and Food Technology, Faculty of Bioenvironmental

13 Science, Kyoto Gakuen University, 1-1 Nanjo-Ohtani, Sogabe, Kameoka 621-8555, 14 Japan.

15

16 *To whom correspondence should be addressed.

17 Phone: +8175753 6387. Fax: +8175753 6454. E-mail: yury@kais.kyoto-u.ac.jp 


\section{Abstract}

20 A number of pink-pigmented facultative methylotrophs (PPFMs) belonging to Methylobacterium spp. isolated from living plant samples were found to require B vitamins for their growth in minimal medium, and most B vitamin-auxotrophic PPFMs required pantothenate (vitamin $B_{5}$ ). Further investigation of pantothenate auxotrophy using the representative strain Methylobacterium sp. OR01 demonstrated that this strain cannot synthesize $\beta$-alanine, one of the precursors of pantothenate. $\beta$-alanine and several precursors of pantothenate restored the growth of Methylobacterium sp. OR01 in minimal medium. Furthermore, this strain could colonize leaves of Arabidopsis thaliana cultivated in medium without pantothenate or its precursors. Pantothenate, $\beta$-alanine and several precursors were detected in the suspension of $A$. thaliana leaves. These results suggest that pantothenate-auxotrophic PPFMs can symbiotically colonize the surface of plant leaves by acquiring $\beta$-alanine and other precursors, in addition to pantothenate. Finally, the fitness advantage of B vitamin auxotrophy of PPFMs in the phyllosphere environment is discussed.

Key words: B vitamin auxotrophy, pantothenate, $\beta$-alanine, Methylobacterium, phyllosphere 


\section{Introduction}

The phyllosphere, which comprises the above ground part of terrestrial plants, provides an extensive habitat for microorganisms. In the phyllosphere, plants are expected to supply all of the nutrients and cofactors required for the growth and survival of phyllospheric microorganisms. Plants are known to produce various kinds of volatile organic compounds (VOCs) $[1,2]$. Among them, methanol is abundantly produced from the methyl ester groups of the plant cell wall component pectin. Methanol present on plant leaves can be utilized as carbon sources by a subset of phyllospheric microorganisms, called methylotrophs [3]. Our previous study using a methylotrophic yeast methanol sensor revealed that the methanol concentration present on the leaf surfaces of Arabidopsis thaliana is oscillated in the range of 0-0.2\% (ca., 0-60 mM) [4]. The presence of sugar compounds including glucose on the leaf surface has also been reported [5]. In addition to these compounds, nitrate, methylamine, and amino acids, which can be used as nitrogen sources, and some trace cofactors like vitamins, have also been reported to be present on the leaf surface $[6,7]$.

The leaf surface is the main area of the phyllosphere and it harbors a unique microbial community. The bacterial population on the leaf surface is estimated to be $10^{6}-10^{7}$ cells $/ \mathrm{cm}^{2}$, while archaea and fungi also colonize on leaves at smaller population sizes than bacteria [8]. Delmotte et al. reported that the dominant class of phyllosphere bacteria on soybean leaves was Alphaproteobacteria, which represented more than $40 \%$ of the population, and included Sphingomonas and Methylobacterium as the major species [9]. Methylobacterium spp. can utilize methanol as the sole carbon and energy source and are also called pink-pigmented facultative methylotrophs (PPFMs). These bacteria are known to have the ability to promote plant growth through the production of phytohormones, such as auxins and cytokinins, and induce systemic resistance against pathogens and diseases [10-14]. Thus, much attention has 
recently been paid to the symbiotic interaction between Methylobacterium spp. and plants, and their potential applications.

The ability of Methylobacterium spp. to grow on methanol is thought to be one of the reasons why these bacteria can dominate on leaf surfaces. In previous studies, the relationship between the ability to grow on methanol and the ability to colonize plant leaf surfaces was investigated using the representative model strain Methylobacterium extorquens AM1, which was originally isolated as an airborne contaminant growing on methylamine [15] and has recently been re-classified as Methylorubrum extorquens [16]. Competition experiments with wild-type and mutant strains of M. extorquens AM1 revealed that mutant strains lacking $m x a F$ or $x o x F$, encoding the large subunit of the $\mathrm{Ca}^{2+}$-dependent methanol dehydrogenase (MDH) or a lanthanide-dependent $\mathrm{MDH}$, respectively, are less competitive than the wild-type strain during colonization on plant leaves $[17,18]$. These findings suggest that the ability to utilize methanol as a carbon source is advantageous for Methylobacterium spp. for proliferation and survival in the phyllosphere.

So far, a number of PPFMs have been isolated from various living plant samples [19-23]. We have investigated the distribution of PPFMs on the leaves and seeds of various vegetables, and found that red perilla plants harbor a dominant population of PPFMs, with Methylobacterium sp. OR01 as a representative strain [24]. We also confirmed direct transmission of Methylobacterium sp. OR01 from red perilla seeds to their leaves, and this strain exhibited a greater ability to colonize on the red perilla plant than M. extorquens AM1 [25]. In general, media for screening or enrichment culture of PPFMs contain a mixture of B vitamins [26-28]. However, our knowledge of the auxotrophic requirements for vitamins and other cofactors by various Methylobacterium spp. is limited. It was reported that the addition of pantothenate (vitamin $\mathrm{B}_{5}$ ) enhanced the growth of several strains of Methylobacterium spp. [29], but there have been no reports whether pantothenate auxotrophy is relevant to the growth environment in the phyllosphere. 
In this study, we found that many PPFMs isolated from living plant samples

94 exhibit B-vitamin auxotrophy, especially pantothenate auxotrophy, and the representative Methylobacterium sp. OR01 could grow on the leaf surface of the model

96 plant Arabidopsis thaliana. The availability of biosynthetic precursors of pantothenate

97 was predicted from the draft genome sequence of Methylobacterium sp. OR01, and we

98 demonstrated that the pantothenate auxotrophic Methylobacterium sp. OR01 can utilize

99 pantothenate or its precursors present on the surface of Arabidopsis leaves. Finally, a

100 fitness advantage of the pantothenate auxotrophic PPFMs compared to the

101 non-auxotrophic $M$. extorquens AM1 in the phyllosphere environment was identified

102 and discussed. 
Bacterial strains and culture conditions. Methylobacterium sp. OR01 [25], M. extorquens AM1 [15], and other Methylobacterium spp. strains isolated from plant samples (Table 1; details of the screening procedure and characterization of each strain will be described elsewhere) were grown at $28^{\circ} \mathrm{C}$ in Hypho minimal medium (MM) [30] supplemented with $0.5 \%(\mathrm{v} / \mathrm{v})$ methanol or $0.2 \%(\mathrm{w} / \mathrm{v})$ disodium succinate as the carbon source. Single B-group vitamins or B-vitamin mixtures (VB mix) were added to MM. The B-group vitamins were thiamine $\left(B_{1}\right)$, niacin $\left(B_{3}\right)$, Ca-pantothenate $\left(B_{5}\right)$, pyridoxine $\mathrm{HCl}\left(\mathrm{B}_{6}\right)$, biotin $\left(\mathrm{B}_{7}\right)$, cobalamin $\left(\mathrm{B}_{12}\right)$, inositol $(\mathrm{B}-\mathrm{h})$, and $p$-amino benzoate (B-x); concentrations in the medium were $4 \mu \mathrm{g} / \mathrm{mL}, 4 \mu \mathrm{g} / \mathrm{mL}, 4 \mu \mathrm{g} / \mathrm{mL}, 2 \mu \mathrm{g} / \mathrm{mL}, 20$ $\mathrm{ng} / \mathrm{mL}, 4 \mu \mathrm{g} / \mathrm{mL}, 2 \mu \mathrm{g} / \mathrm{mL}$, and $2 \mu \mathrm{g} / \mathrm{mL}$, respectively.

Measurement of populations of PPFMs on Arabidopsis thaliana leaves.

Methylobacterium sp. OR01 and M. extorquens AM1 were grown in MM containing $0.5 \%$ methanol and VB mix at $28^{\circ} \mathrm{C}$ for 3 days. The bacterial cultures were collected and washed twice with sterilized water and then resuspended in sterilized water to obtain cell suspension with an $\mathrm{OD}_{600}$ of 0.1 .

Arabidopsis seeds were sterilized with $70 \%$ ethanol for $30 \mathrm{sec}$ followed by $0.5 \%$ hypochlorite solution for $15 \mathrm{~min}$. They were mixed with the cell suspension and gently shaken by using a rotating mixer (Rotator RT-5, Taitec, Saitama, Japan) for $2 \mathrm{~h}$. Seeds inoculated with Methylobacterium sp. OR01 or M. extorquens AM1 were placed on agar plate medium, Murashige and Skoog medium (Duchefa, Haarlem, Netherlands) supplied with $0.8 \%$ Bacto agar (Difco Becton Dickinson, Franklin Lakes, NJ, USA), which did not contain pantothenate or its precursors. The seeds were aseptically incubated in a plant growth chamber (Nippon Medical \& Chemical Instruments, Osaka, Japan) at $25^{\circ} \mathrm{C}$ for 14 days in a daily light/dark cycle $(14 \mathrm{~h}$ light $/ 10 \mathrm{~h}$ dark). The 
fourth leaves were removed from the plants and soaked in $500 \mu \mathrm{L}$ of sterilized water.

Bacterial cells colonizing on the leaf surface were removed from the leaves by vortexing for $2 \mathrm{~min}$ and incubating in an ultrasonic bath (UT205S, Sharp, Osaka, Japan) for $15 \mathrm{~min}$. The supernatant was serially diluted and plated onto MM agar medium containing $0.5 \%$ methanol and VB mix. Plates were incubated at $28^{\circ} \mathrm{C}$ for $4-5$ days and then the number of pink colonies was counted and the colony forming units (CFU/leaf) were determined.

Quantitative analysis of compounds on the leaf surface. After A. thaliana was grown on Murashige and Skoog agar medium lacking pantothenate in a plant growth chamber at $25^{\circ} \mathrm{C}$ for 14 days, ten leaves were separated from plants and the cut edges were sealed with instant glue (Aron Alpha EXTRA, Toagosei, Tokyo, Japan) to prevent compounds from flowing out of leaves. These leaves were soaked in $500 \mu \mathrm{L}$ of sterilized water for $10 \mathrm{~min}$ to collect compounds present on the leaf surface. The amount of pantothenate, spermine, spermidine, 5,6-dihydrouracil, $N$-carbamoyl- $\beta$-alanine, and 3-hydroxypropanoate in the collected solution was measured by LC-MS/MS analysis. The collected solution was subjected to chromatographic separation on a Hydrosphere C18 column (column size $2 \times 150 \mathrm{~mm}$, YMC, Kyoto, Japan) with a Prominence UFLC HPLC system (Shimazu, Kyoto, Japan). The mobile phase used was $\mathrm{H}_{2} \mathrm{O}$ containing $0.05 \%$ acetic acid (solvent $\mathrm{A}$ ) and methanol (solvent B). The gradient conditions for B\% were: $0-6.0 \mathrm{~min}=2 \%, 6.0-10.0 \mathrm{~min}=$ $2-80 \%, 10.0-12.0 \min =80-2 \%, 12.0-20.0 \min =2 \%$ for $N$-carbamoyl- $\beta$-alanine, 3-hydroxypropanoate, and pantothenate analysis, and 0-10.0 $\mathrm{min}=2 \%$ for spermine, spermidine, and 5,6-dihydrouracil analysis. The flow rate was $0.1 \mathrm{~mL} / \mathrm{min}$ and the injection volume was $5 \mu \mathrm{L}$. carbamate (APDS) regent (Amino Acid Analysis Reagent, FUJIFILM Wako Pure 
157 Chemical, Osaka, Japan) and subjected to chromatographic separation on a Wakopak®

158 Wakosil-II 3C8-100HG column (column size 2 x 100 mm, FUJIFILM Wako Pure

159 Chemical) [31]. The mobile phase used was APDSTAG Wako Eluent (solvent A,

160 FUJIFILM Wako Pure Chemical) and 60\% acetonitrile solution (solvent B). The

161 gradient conditions for $\mathrm{B} \%$ were: $0-1.25 \mathrm{~min}=4 \%, 1.25-1.26 \mathrm{~min}=4-15 \%, 1.26-5.0$

$162 \min =15-20 \%, 5.0-5.5 \min =20-50 \%, 5.5-6.5 \min =50-95 \%, 6.5-6.75 \min =95 \%$,

$1636.75-6.76 \mathrm{~min}=95-4 \%, 6.76-12.0 \mathrm{~min}=4 \%$. The flow rate was $0.3 \mathrm{~mL} / \mathrm{min}$ and the

164 injection volume was $2 \mu \mathrm{L}$.

165 Detection was conducted with a 4000 QTrap with turboionspray source (AB

166 Sciex, Framingham, MA, USA). Data acquisition for all experiments was carried out

167 by Multiple Reaction Monitoring (MRM) method operated with Analyst software ver.

1681.5 (AB Sciex). The setting parameters were shown in Table S1.

169 


\section{Results}

\section{Pantothenate auxotrophy of PPFMs isolated from living plant samples}

Of the 13 tested strains that were isolated from living plant samples, 10 strains (By, B2B, RS1, C06, C16, C17, In1, OR01, Sh, and Rst) required the B-group vitamins mixture (VB mix) for their growth on Hypho minimal medium (MM), and the other 3 strains (C1, Dw, and Rfw) did not (Table 1). Next we investigated which B-vitamins could recover the growth of each strain on MM. Results showed that the addition of pantothenate (vitamin $\mathrm{B}_{5}$ ) restored the growth of 9 strains on MM (Table 1). The 16S rRNA gene sequence analysis revealed that these 9 strains belong to M. radiotolerans and M. fusisawaense (isolation and identification of these strains will be described elsewhere). Methylobacterium sp. Rst required both biotin (vitamin $\mathrm{B}_{7}$ ) and pyridoxine (vitamin $\mathrm{B}_{6}$ ) for its growth on $\mathrm{MM}$. These results indicate that many Methylobacterium spp. that exhibit B-group vitamins auxotrophy, could inhabit the phyllosphere.

Biosynthetic pathway for pantothenate in Methylobacterium sp. OR01

Because the pantothenate auxotrophs were closely related to each other, further investigation of pantothenate auxotrophy was conducted using the representative strain Methylobacterium sp. OR01, which was isolated from red perilla seeds [25] and whose draft genome sequence was determined (details will be described elsewhere).

Pantothenate is synthesized by a condensation reaction of pantoate and $\beta$-alanine in bacteria, by pantothenate synthetase (EC: 6.3.2.1) encoded by the panC gene (Figure 1(a)) [32]. First, we examined whether Methylobacterium sp. OR01 required pantoate or $\beta$-alanine for its growth in minimal medium. Methylobacterium sp. OR01 was able to grow in MM supplemented with $\beta$-alanine, although the maximum $\mathrm{OD}_{600}$ of this strain when supplemented with $\beta$-alanine was lower than that with pantothenate. In 
contrast, Methylobacterium sp. OR01 did not grow with pantoate (Figure 1(b)). These results indicate that the pantothenate auxotrophy of Methylobacterium sp. OR01 is due to the inability to synthesize $\beta$-alanine.

Several biosynthetic pathways for $\beta$-alanine are known in bacteria, in which $\beta$-alanine is synthesized from L-aspartate, putrescine, uracil, $\alpha$-alanine, malonate, oxalate, and lactate (Figure 2) $[33,34]$. To identify the defective portion of the $\beta$-alanine biosynthetic pathways in Methylobacterium sp. OR01, genes encoding putative enzymes involved in $\beta$-alanine biosynthesis were searched in the draft genome sequence. In the pathways from uracil and putrescine, homologs of $\beta$-alanine biosynthetic enzyme genes were found in Methylobacterium sp. OR01 (red arrows in Figure 2). It is noteworthy that Methylobacterium sp. OR01 has homologs of genes for all enzymes in the $\beta$-alanine biosynthetic pathway from uracil via 3-oxopropanoic acid, although this strain cannot synthesize $\beta$-alanine. Amino acid sequences of the genes annotated as enzymes involved in the pathway from uracil to $\beta$-alanine in Methylobacterium sp. OR01 exhibited more than $60 \%$ similarity with those characterized in E. coli or Pseudomonas aeruginosa (data not shown). It is possible that enzymes in Methylobacterium sp. OR01 show different substrate specificity or these genes are not expressed in this strain.

Next, to identify precursors of $\beta$-alanine that recover the growth of Methylobacterium sp. OR01 in minimal medium, the strain was cultured in MM supplemented with 12 different compounds, lactate, L-aspartate, spermine, spermidine, putrescine, uracil, 5,6-dihydrouracil, $N$-carbamol- $\beta$-alanine, $\alpha$-alanine, malonate, oxalate, and 3-hydroxypropanoate (Figure 2). Among them, addition of spermine, spermidine, 5,6-dihydrouracil, $N$-carbamol- $\beta$-alanine, and 3-hydroxypropanoate could recover the growth of Methylobacterium sp. OR01 in MM (Figure 3). The growth yields of Methylobacterium sp. OR01 supplemented with spermine or spermidine were lower than that with 5,6-dihydrouracil, $N$-carbamol- $\beta$-alanine, or 3-hydroxypropanoate. 
224 On the other hand, all starting compounds for $\beta$-alanine biosynthesis, including

225 L-aspartate, putrescine, uracil, $\alpha$-alanine, malonate, oxlate, and lactate, could not recover the growth of Methylobacterium sp. OR01 on MM. Judging from these results, Methylobacterium sp. OR01 can utilize $\beta$-alanine in addition to spermine, spermidine, 5,6-dihydrouracil, N-carbamoyl- $\beta$-alanine, or 3-hydroxypropanoate, as the precursors of pantothenate biosynthesis.

Colonization of pantothenate auxotrophic Methylobacterium sp. OR01 on the surface of Arabidopsis leaves

In our previous study, we revealed that Methylobacterium sp. OR01 is a dominant colonizer on the red perilla plant [24]. Our present results demonstrating that Methylobacterium sp. OR01 could utilize $\beta$-alanine and its precursors suggest that this strain acquires pantothenate or its precursors from the plant surface environment. To confirm that Methylobacterium sp. OR01 acquires pantothenate or its precursors from plants, we inoculated cells of Methylobacterium sp. OR01 on sterilized seeds of the model plant $A$. thaliana and cultivated the plant on medium lacking pantothenate and its precursors. After two-weeks of cultivation, the leaves were suspended in sterilized water and sonicated to release bacterial cells from the plant surface, and the colony forming units (CFU/leaf) of the cell suspension were determined as described in Materials and Methods. M. extorquens AM1, a pantothenate prototroph and model Methylobacterium sp. strain was used for comparison (Table 1). The suspension from

245 leaves inoculated with Methylobacterium sp. OR01 contained 7.9 x $10^{4}\left(2.8 \times 10^{4}-1.8\right.$ x $\left.10^{5}\right) \mathrm{CFU} /$ leaf, which was comparable to that with M. extorquens AM1, $2.7 \times 10^{4}(1.0$

$247 \times 10^{4}-7.8 \times 10^{4}$ ) CFU/leaf (Figure 4(a)). These results indicate that the

248 pantothenate-auxotrophic Methylobacterium sp. OR01 acquired sufficient pantothenate or its precursor compounds to allow proliferation during colonization on Arabidopsis plant. In addition, the dominant colonization of Methylobacterium sp. OR01 over M. 
extorquens AM1 on the Arabidopsis leaves was observed as shown in Figure 4(b) (see Discussion).

Pantothenate and its precursors are present on the surface of Arabidopsis leaves To investigate whether a sufficient amount of pantothenate and/or its precursors to compensate for the pantothenate auxotrophy of Methylobacterium sp. OR01 is present on the plant leaf surface, we used LC-MS/MS analysis to quantify the amounts of pantothenate, $\beta$-alanine and its precursors, spermine, spermidine, 5,6-dihydrouracil, $N$-carbamoyl- $\beta$-alanine, and 3-hydroxypropanoate, which were washed off of the surface of Arabidopsis leaves into the leaf-suspension solution. The presence of significant amounts of pantothenate, $\beta$-alanine, spermidine, $N$-carbamoyl- $\beta$-alanine, and 3-hydroxypropanoate were detected, while spermine and 5,6-dihydrouracil were below the limit of detection (Table 2$)$. The amount of $\beta$-alanine $\left(1.1 \times 10^{3} \mathrm{pmol} / \mathrm{g}\right.$ fresh leaves) was c.a. 100 -fold greater than that of pantothenate $\left(1.5 \times 10^{1} \mathrm{pmol} / \mathrm{g}\right.$ fresh leaves) and much higher than that of $\beta$-alanine precursors. Judging from these results, the pantothenate auxotrophic Methylobacterium sp. OR01 appears to acquire pantothenate from the plant and also acquires $\beta$-alanine for use in pantothenate biosynthesis.

\section{Ability of Methylobacterium sp. OR01 to utilize pantotheanate and $\beta$-alanine}

To predict which compounds, pantothenate or $\beta$-alanine, contribute to the growth of Methylobacterium sp. OR01 in the phyllosphere, we investigated the ability of this strain to utilize pantothanate and $\beta$-alanine. Methylobacterium sp. OR01 was cultivated in MM supplemented with various concentrations of pantothanate or $\beta$-alanine. Methylobacterium sp. OR01 could grow in the presence of more than $1 \mu \mathrm{M}$ pantothenate or more than $5 \mu \mathrm{M} \beta$-alanine (Figure 5). The maximum growth rate was observed when the medium was supplemented with $5 \mu \mathrm{M}$ pantothenate or $45 \mu \mathrm{M}$ 
$278 \beta$-alanine. Thus, the concentration of pantothenate that supported the optimal growth 279 of Methylobacterium sp. OR01 was c.a. 10-fold lower than that of $\beta$-alanine. However, 280 since the concentration of $\beta$-alanine on the leaf surface was 100 -fold higher than that of 281 pantothenate, Methylobacterium sp. OR01 is thought to utilize both pantothenate and $282 \beta$-alanine in the phyllosphere.

283 
Phyllosphere microorganisms utilize various nutrient compounds present on plant leaves for their growth and survival in the phyllosphere. For example, methanol and sugar compounds, including glucose, can be utilized as carbon sources, and nitrate and methylamine can be utilized as nitrogen sources [3, 5, 6, 35]. However, it was unknown how phyllosphere microorganisms utilize low molecular compounds that function as cofactors or vitamins in the phyllosphere. In this study, we focused on B vitamin auxotrophy of Methylobacterium spp., which are known to be the dominant colonizers in the phyllosphere, and revealed that a number of Methylobacterium strains isolated from living plant samples required B vitamins, especially pantothenate (vitamin $\mathrm{B}_{5}$ ), for their growth.

According to the KEGG pathway database (https://www.genome.jp/kegg/pathway.html), all enzymes involved in pantothenate biosynthesis except ketopantoate reductase, which generates pantoate from ketopantoate, are encoded in the genome of Arabidopsis, soybean, rice, tomato, and potato [36]. Generation of pantoate from ketopantoate is thought to be catalyzed not by ketopantoate reductase, but by acetohydroxy acid isomeroreductase encoded by the $i l v C$ gene in $A$. thaliana [37]. Therefore, plants are assumed to be able to synthesize pantothenate. It was also reported that not only pantothenate but also other B vitamins, including thiamine and pyridoxine, are present on leaves of Sambucus nigra [7]. Therefore, B vitamin auxotrophs living in the phyllosphere are assumed to utilize B vitamins synthesized by plants. Indeed, pantothenate auxotrophic Methylobacterium sp. OR01 was shown to utilize pantothenate or its precursors from the leaf surface environment of Arabidopsis (Figure 4(a) and Table 2).

The growth of the pantothenate auxotrophic Methylobacterium sp. OR01 on minimal medium was recovered by the addition of $\beta$-alanine, one of the precursors of 
311 pantothenate biosynthesis (Figure 1(b)). Several putative genes encoding enzymes

312 involved in $\beta$-alanine biosynthesis were found in the draft genome sequence of Methylobacterium sp. OR01 (Figure 2), and spermine, spermidine, 5,6-dihydrouracil, $N$-carbamoyl- $\beta$-alanine, and 3-hydroxypropanoate were found to restore the ability of Methylobacterium sp. OR01 to grow in minimal medium (Figure 3). These results suggest that multiple pathways can function in $\beta$-alanine biosynthesis in Methylobacterium sp. OR01. The ability of the pantothenate auxotrophic strain to synthesize $\beta$-alanine not from a single compound but from several different compounds may enable this strain to adapt to various environments including the phyllosphere.

We demonstrated that the pantothenate auxotrophic Methylobacterium sp. OR01 could colonize Arabidopsis leaves cultivated on plant medium which did not contain pantothenate or its precursors (Figure 4(a)) and that pantothenate, $\beta$-alanine, and several precursor compounds were present on Arabidopsis leaves (Table 2). These results suggest that Methylobacterium sp. OR01 can acquire pantothenate and/or its precursors produced by host plants. We also revealed that the amount of $\beta$-alanine on Arabidopsis leaves was ca. 100-fold higher than that of pantothenate and other precursors (Table 2) and that the minimum concentrations of pantothenate and $\beta$-alanine necessary to support the growth of Methylobacterium sp. OR01 in minimal medium were almost the same (Figure 5). The concentrations of pantothenate and $\beta$-alanine on the leaf surface cannot be estimated by the values indicated in Table 2, however, enough amount to support the growth of bacterial cells is thought to be constantly supplied in the phyllosphere. Therefore, not only pantothenate but also $\beta$-alanine produced by the host plant, could support the proliferation of the pantothenate auxotrophic Methylobacterium sp. OR01 on plant surfaces.

Regarding the uptake of pantothenate and $\beta$-alanine by Methylobacterium sp. 39], were not found in the draft genome sequence of Methylobacterium sp. OR01. 
However, a gene encoding a putative amino acid permease, which has $43 \%$ amino acid sequence similarity to the $\beta$-alanine transporter CycA of E. coli [40], was found, and might be involved in uptake of pantothenate.

In our previous study, the red perilla colonization ability of Methylobacterium sp. OR01, which was originally isolated from red perilla seeds, was compared with that of M. extorquens AM1 [25]. Sterilized red perilla seeds were inoculated with Methylobacterium sp. OR01, M. extorquens AM1, or a mixture of both strains and the CFUs in the suspension of leaves were determined after two-weeks of cultivation of the red perilla plant. Although both strains colonized red perilla leaves when inoculated separately onto the seeds, only Methylobacterium sp. OR01 colonized leaves when the mixture was inoculated [25]. In this study, we conducted the same experiments using the model plant A. thaliana, and the presence of Methylobacterium sp. OR01 alone on leaves was confirmed in the case of the mixed inoculation with Methylobacterium sp. OR01 and M. extorquens AM1 (Figure 4(b)). As revealed in the present study, Methylobacterium sp. OR01 is a pantothenate auxotroph and M. extorquens AM1 is prototrophic for not only pantothenate but also other B vitamins (Table 1). Under the tested conditions, the pantothenate auxotrophic Methylobacterium sp. OR01 dominated over the non-auxotrophic strain on plant leaves.

One reason why the auxotrophic strain was more effective at colonizing than the prototrophic strain might be due to that the auxotrophic strain does not need to consume energy for the synthesis of pantothenate or its precursors. Biosynthesis of one molecule of pantothenate requires 2 molecules of NADPH for generation of panthoate and one molecule of ATP for the condensation reaction between panthoate and $\beta$-alanine. Furthermore, the biosynthesis of one molecule of $\beta$-alanine from uracil requires one molecule of NADH. We also found that Methylobacterium sp. strain Rst is an auxotroph for biotin and pyridoxine (Table 1). Pyridoxine biosynthesis from pyridoxal requires 1 molecule of NADPH, and biotin biosynthesis from pimelic acid 
requires 2 molecules of ATP.

Most strains of intestinal bacteria are known to be auxotrophs. For example, the lactic acid bacterium, Leuconostoc citrovorum, requires many amino acids, nucleobases, and vitamins for growth in synthetic medium [41]. Using co-culture experiments with the mutant and wild-type strains of E. coli and Acinetobacter baylyi, D'Souza et al. demonstrated that the loss of essential biosynthetic genes for a certain amino acids, nucleobases, or vitamins was generally beneficial when the required compounds were present in the growth environment [42]. In other words, the fitness advantage of the auxotrophic mutant increased more than that of the prototrophic wild type. As with the case of bacteria living in the intestinal nutrient environment, Methylobacterium sp. OR01, which is a dominant colonizer on plant leaves and can be directly transmitted from seeds to leaves [25], might have increased fitness by acquiring pantothenate and $\beta$-alanine, which are present in the phyllosphere, saving energy costs for biosynthesis of these compounds, and by possessing multiple pathways for $\beta$-alanine biosynthesis from several precursors available in the phyllosphere.

\section{Author contributions}

Y.Y. and H.I. performed the experiments. Y.Y., H.I., Y.S., and H.Y. designed the experiments and analyzed the data. Y.Y., Y.S., and H.Y. wrote the manuscript.

\section{Acknowledgement}

This research was supported in part by a Grant-in-Aid for Scientific Research (B) (16H02997 to H.Y.) from the Japan Society for the Promotion of Science. It was also supported in part by Advanced Low Carbon Technology Research and Development Program (ALCA) from Japan Science and Technology Agency (Y.S.). 


\section{References}

392

[1] Seco R, Penuelas J, and Filella I. Short-chain oxygenated VOCs: Emission and uptake by plants and atmospheric sources, sinks, and concentrations. Atmos Environ. 2007;41:2477-2499.

[3] Galbally I, and Kirstine W. The production of methanol by flowering plants and the global cycle of methanol. J Atmos Chem. 2002;43:195-229.

[4] Kawaguchi K, Yurimoto H, Oku M, et al. Yeast methylotrophy and autophagy in a methanol-oscillating environment on growing Arabidopsis thaliana leaves. PLoS One. 2011;6:e25257.

[5] Mercier J, and Lindow SE. Role of leaf surface sugars in colonization of plants by bacterial epiphytes. Appl Environ Microbiol. 2000;66:369-374.

[6] Shiraishi K, Oku M, Kawaguchi K, et al. Yeast nitrogen utilization in the phyllosphere during plant lifespan under regulation of autophagy. Sci Rep. 2015;5:9719.

[7] Gargallo-Garriga A, Sardans J, Perez-Trujillo M, et al. Shifts in plant foliar and floral metabolomes in response to the suppression of the associated microbiota. BMC Plant Biol. 2016;16:78.

[8] Lindow SE, and Brandl MT. Microbiology of the phyllosphere. Appl Environ Microbiol. 2003;69:1875-1883.

416 [10] Senthilkumar M, Madhaiyan M, Sundaram S, et al. Intercellular colonization and growth promoting effects of Methylobacterium sp. with plant-growth regulators on rice (Oryza sativa L. Cv CO-43). Microbiol Res. 2009;164:92-104. 
[11] Hornschuh M, Grotha R, and Kutschera U. Moss-associated methylobacteria as phytosymbionts: an experimental study. Naturwissenschaften. 2006;93:480-486.

[12] Madhaiyan M, Poonguzhali S, Ryu J, et al. Regulation of ethylene levels in canola (Brassica campestris) by 1-aminocyclopropane-1-carboxylate deaminase-containing Methylobacterium fujisawaense. Planta. 2006;224:268-278.

[13] Koenig RL, Morris RO, and Polacco JC. tRNA is the source of low-level trans-zeatin production in Methylobacterium spp. J Bacteriol. 2002;184:1832-1842.

[14] Ardanov P, Sessitsch A, Haggman H, et al. Methylobacterium-induced endophyte community changes correspond with protection of plants against pathogen attack. PLoS One. 2012;7:e46802.

[15] Peel D, and Quayle JR. Microbial growth on C1 compounds. I. Isolation and characterization of Pseudomonas AM1. Biochem J. 1961;81:465-469.

[16] Green PN, and Ardley JK. Review of the genus Methylobacterium and closely related organisms: a proposal that some Methylobacterium species be reclassified into a new genus, Methylorubrum gen. nov. Int J Syst Evol Microbiol. 2018;68:2727-2748.

[17] Sy A, Timmers AC, Knief C, et al. Methylotrophic metabolism is advantageous for Methylobacterium extorquens during colonization of Medicago truncatula under competitive conditions. Appl Environ Microbiol. 2005;71:7245-7252.

[18] Schmidt S, Christen P, Kiefer P, et al. Functional investigation of methanol dehydrogenase-like protein XoxF in Methylobacterium extorquens AM1. Microbiology. 2010;156:2575-2586.

[19] Van Aken B, Peres CM, Doty SL, et al. Methylobacterium populi sp. nov., a novel aerobic, pink-pigmented, facultatively methylotrophic, methane-utilizing bacterium isolated from poplar trees (Populus deltoides x nigra DN34). Int J 
Syst Evol Microbiol. 2004;54:1191-1196.

[20] Madhaiyan M, Kim BY, Poonguzhali S, et al. Methylobacterium oryzae sp. nov., an aerobic, pink-pigmented, facultatively methylotrophic, 1-aminocyclopropane-1-carboxylate deaminase-producing bacterium isolated from rice. Int J Syst Evol Microbiol. 2007;57:326-331.

[21] Schauer S, and Kutschera U. A novel growth-promoting microbe, Methylobacterium funariae sp. nov., isolated from the leaf surface of a common moss. Plant Signal Behav. 2011;6:510-515.

[22] Sy A, Giraud E, Jourand P, et al. Methylotrophic Methylobacterium bacteria nodulate and fix nitrogen in symbiosis with legumes. J Bacteriol. 2001;183:214-220.

[23] Raja P, Balachandar D, and Sundaram S. Genetic diversity and phylogeny of pink-pigmented facultative methylotrophic bacteria isolated from the phyllosphere of tropical crop plants. Biol Fertil Soils. 2008;45:45-53.

[24] Mizuno M, Yurimoto H, Yoshida N, et al. Distribution of pink-pigmented facultative methylotrophs on leaves of vegetables. Biosci Biotechnol Biochem. 2012;76:578-580.

[25] Mizuno M, Yurimoto H, Iguchi H, et al. Dominant colonization and inheritance of Methylobacterium sp. strain OR01 on perilla plants. Biosci Biotechnol Biochem. 2013;77:1533-1538.

[26] Tani A, Sahin N, and Kimbara K. Methylobacterium oxalidis sp. nov., isolated from leaves of Oxalis corniculata. Int J Syst Evol Microbiol. 2012;62:1647-1652.

[27] Shen PH, and $\mathrm{Wu}$ B. Over-expression of a hydroxypyruvate reductase in Methylobacterium sp. MB200 enhances glyoxylate accumulation. J Ind Microbiol Biotechnol. 2007;34:657-663.

[28] Tani A, Sahin N, Matsuyama Y, et al. High-throughput identification and 
screening of novel Methylobacterium species using whole-cell MALDI-TOF/MS analysis. PLoS One. 2012;7:e40784.

[29] Urakami T, Araki H, Suzuki K-I, et al. Further studies of the genus Methylobacterium and description of Methylobacterium aminovorans sp. nov. Int J Syst Evol Microbiol. 1993;43:504-513.

[30] Delaney NF, Kaczmarek ME, Ward LM, et al. Development of an optimized medium, strain and high-throughput culturing methods for Methylobacterium extorquens. PLoS One. 2013;8:e62957.

[31] Shimbo K, Oonuki T, Yahashi A, et al. Precolumn derivatization reagents for high-speed analysis of amines and amino acids in biological fluid using liquid chromatography/electrospray ionization tandem mass spectrometry. Rapid Commun Mass Spectrom. 2009;23:1483-1492.

[32] Miyatake K, Nakano Y, and Kitaoka S. Pantothenate synthetase from Escherichia coli [D-pantoate: $\beta$-alanine ligase (AMP-forming), EC 6.3.2.1]. Methods Enzymol. 1979;62:215-219.

[33] Herrmann G, Selmer T, Jessen HJ, et al. Two beta-alanyl-CoA: ammonia lyases in Clostridium propionicum. FEBS J. 2005;272:813-821.

[34] Wang Y, Xu H, and White RH. $\beta$-Alanine biosynthesis in Methanocaldococcus jannaschii. J Bacteriol. 2014;196:2869-2875.

[35] Gruffaz C, Muller EE, Louhichi-Jelail Y, et al. Genes of the $N$-methylglutamate pathway are essential for growth of Methylobacterium extorquens DM4 with monomethylamine. Appl Environ Microbiol. 2014;80:3541-3550.

[36] Coxon KM, Chakauya E, Ottenhof $\mathrm{HH}$, et al. Pantothenate biosynthesis in higher plants. Biochem Soc Trans. 2005;33:743-746.

[37] Primerano DA, and Burns RO. Role of acetohydroxy acid isomeroreductase in biosynthesis of pantothenic acid in Salmonella typhimurium. J Bacteriol. $1983 ; 153: 259-269$. 
500 [38] Rodionov DA, Hebbeln P, Eudes A, et al. A novel class of modular transporters

501

502

503

504

505

506

507

508

509

510

511

512

513 for vitamins in prokaryotes. J Bacteriol. 2009;191:42-51.

[39] Vallari DS, and Rock CO. Isolation and characterization of Escherichia coli pantothenate permease (panF) mutants. J Bacteriol. 1985;164:136-142.

[40] Schneider F, Krämer R, and Burkovski A. Identification and characterization of the main $\beta$-alanine uptake system in Escherichia coli. Appl Microbiol Biotechnol. 2004;65:576-582.

[41] Sauberlich HE, and Baumann CA. A factor required for the growth of Leuconostoc citrovorum. J Biol Chem. 1948;176:165-173.

[42] D'Souza G, Waschina S, Pande S, et al. Less is more: selective advantages can explain the prevalent loss of biosynthetic genes in bacteria. Evolution. 2014;68:2559-70. 


\section{Figure legends}

515

516

517

518

519

520

521

522

523

524

525

526

527

528

529

530

531

532

533

534

535

536

537

538

539

540

541

Figure 1. $\beta$-Alanine auxotrophy of Methylobacterium sp. OR01.

(a) Biosynthesis of pantothenic acid. Pantothenic acid is synthesized by a condensation reaction of pantoic acid and $\beta$-alanine. The panC encodes pantothenate synthetase (EC: 6.3.2.1). (b) Growth of Methylobacterium sp. OR01.

Methylobacterium sp. OR01 was cultured in MM containing methanol as the sole carbon source, supplemented with $15 \mu \mathrm{M}$ each of pantothenate (open circles), $\beta$-alanine (closed circles) or pantoate (open squares).

Figure 2. Pathways for bacterial $\beta$-alanine biosynthesis.

Red arrows indicate that homologous genes for enzymes involved in $\beta$-alanine biosynthesis were found in the genome of Methylobacterium sp. OR01. Black arrows indicate that the homologous gene was absent in the genome. Black frames indicate compounds that could not restore the growth of Methylobacterium sp. OR01; Red frames indicate compounds that could restore the growth of Methylobacterium sp. OR01 (Figure 3).

Figure 3. Growth of Methylobacterium sp. OR01 supplemented with $\beta$-alanine precursors.

Methylobacterium sp. OR01 was cultivated in MM containing methanol as a carbon source and supplemented with $60 \mu \mathrm{M}$ of each $\beta$-alanine precursor. The $\mathrm{OD}_{600}$ values after cultivation for $72 \mathrm{~h}$ are shown.

Figure 4. Population of PPFMs on the Arabidopsis leaves.

(a) The sterilized seeds of A. thaliana were inoculated with Methylobacterium sp. OR01 or M. extorquens AM1 and sowed onto Murashige and Skoog agar medium without pantothenate. The plants were grown for 14 days and the fourth leaves were 
removed from the plant and suspended in sterilized water. CFUs of the cell suspension were measured as described in Materials and Methods. Box $=25$ th and 75th percentiles; bars $=$ minimum or maximum values (if the minimum value is lower than the 25 th percentiles $-1.5 \mathrm{x}$ box height or maximum value is higher than the 75 th percentile $+1.5 \mathrm{x}$ box height, then bars $=25$ th percentile $-1.5 \mathrm{x}$ box height and 75 th percentile $+1.5 \mathrm{x}$ box height). Dot plot describes the CFUs/leaf for the individual leaves. Methylobacterium sp. OR01, n=40; M. extorquens AM1, n=42. (b) The sterilized seeds of $A$. thaliana were inoculated with a mixture of Methylobacterium sp. OR01 $\left(\mathrm{Km}^{\mathrm{r}}\right)$ and M. extorquens AM1 $\left(\mathrm{Tet}^{\mathrm{r}}\right)$ [25], and sowed onto Murashige and Skoog agar medium without pantothenate. The plants were grown for 14 days and the fourth leaves were removed from the plant and suspended in sterilized water. CFUs of the cell suspension were measured as described in Materials and Methods using MM agar plates containing kanamycin $(20 \mu \mathrm{g} / \mathrm{mL})$ or tetracycline $(10 \mu \mathrm{g} / \mathrm{mL}) . \quad$ Box $=25$ th and 75 th percentiles; bars $=$ minimum or maximum values (if the minimum value is lower than the 25 th percentiles $-1.5 \mathrm{x}$ box height or maximum value is higher than the 75 th percentile $+1.5 \mathrm{x}$ box height, then bars $=25$ th percentile $-1.5 \mathrm{x}$ box height and 75 th percentile $+1.5 \mathrm{x}$ box height). Dot plot describes the CFUs/leaf for the individual leaves. Methylobacterium sp. OR01, n=50; M. extorquens AM1, n=50. n.d., not detected.

Figure 5. Growth of Methylobacterium sp. OR01 in minimal medium supplemented with various concentrations of pantothenate or $\beta$-alanine.

Methylobacterium sp. OR01 was cultivated in MM containing $0.5 \%$ methanol as the carbon source supplemented with (a) pantothenate and (b) $\beta$-alanine. Each concentration of pantothenate and $\beta$-alanine is indicated within the graphs. 
Table 1. Pantothenate auxotrophy of Methylobacterium sp. strains isolated from living plant samples.

\begin{tabular}{|c|c|c|c|c|c|c|}
\hline \multirow[b]{2}{*}{ Strain } & \multirow[b]{2}{*}{ Source } & \multirow[b]{2}{*}{ Closely-related strain } & \multirow{2}{*}{$\begin{array}{c}\text { 16S rRNA } \\
\text { identity (\%) }\end{array}$} & \multicolumn{3}{|c|}{ Growth $^{\mathrm{a}}$} \\
\hline & & & & with VB mix ${ }^{b}$ & without $\mathrm{VB} \mathrm{mix}^{\mathrm{c}}$ & $\begin{array}{c}\text { with } \\
\text { pantothenate }^{\mathrm{d}}\end{array}$ \\
\hline By & Yew leaf & M. radiotolerans strain 91a & 99.7 & + & - & + \\
\hline B2B & Rice leaf & M. radiotolerans strain 91a & 99.8 & + & - & + \\
\hline RS1 & Rice seed & M. fujisawaense strain JoN18 & 100 & + & - & + \\
\hline $\mathrm{C} 06$ & Rice leaf & M. fujisawaense strain JoN18 & 100 & + & - & + \\
\hline $\mathrm{C} 16$ & Rice leaf & M. fujisawaense strain JoN18 & 100 & + & - & + \\
\hline $\mathrm{C} 17$ & Rice leaf & M. fujisawaense strain JoN18 & 99.9 & + & - & + \\
\hline In1 & Yew leaf & M. radiotolerans strain $91 \mathrm{a}$ & 100 & + & - & + \\
\hline OR01 & Red perilla seeds & M. fujisawaense strain JoN18 & 100 & + & - & + \\
\hline Sh & Camellia leaf & M. radiotolerans strain 91a & 99.9 & + & - & + \\
\hline $\mathrm{C} 1$ & Clover leaf & M. extorquens AM1 & 99.9 & + & + & $\overline{+}$ \\
\hline Dw & Duck weed & M. adhaesivum strain $45 \mathrm{i}$ & 100 & + & + & + \\
\hline Rfw & Paddy water & M. populi strain BJ001 & 99.7 & + & + & + \\
\hline Rst & Rice stem & M. platani strain JoF11 & 99.8 & + & - & - \\
\hline AM1 & - & M. extorquens AM1 & 100 & + & + & + \\
\hline
\end{tabular}

a + , growth; -, no growth.

${ }^{\mathrm{b}}$ Each strain was cultured in MM with VB mix. VB mix contains thiamine $\left(\mathrm{B}_{1}\right)$, niacin $\left(\mathrm{B}_{3}\right)$, Ca-pantothenate $\left(\mathrm{B}_{5}\right)$, pyridoxine $\mathrm{HCl}\left(\mathrm{B}_{6}\right)$, biotin $\left(\mathrm{B}_{7}\right)$, cobalamin $\left(\mathrm{B}_{12}\right)$, inositol (B-h), and $p$-amino benzoate $(\mathrm{B}-\mathrm{x})$. Their concentrations are described in Materials and Methods.

${ }^{\mathrm{c}}$ Each strain was cultured in MM without VB mix.

${ }^{\mathrm{d}}$ Each strain was cultured in MM with pantothenate. 
Table 2. Concentration of pantothenate and its precursors in the suspension of the leaves of A. thaliana.

\begin{tabular}{lc}
\hline Compounds & Concentration $^{\mathrm{a}}(\mathrm{pmol} / \mathrm{g}$ fresh leaves $)$ \\
\hline pantothenate & $(1.5 \pm 0.5) \times 10^{1}$ \\
$\beta$-alanine & $(1.1 \pm 0.7) \times 10^{3}$ \\
spermine & n.d. $^{\mathrm{b}}$ \\
spermidine & $(3.9 \pm 1.4) \times 10^{1}$ \\
5,6-dihydrouracil & n.d. \\
$N$-carbamoyl- $\beta$-alanine & $(7.8 \pm 4.3) \times 10^{0}$ \\
3-hydroxypropanoate & $(2.7 \pm 0.4) \times 10^{1}$ \\
\hline
\end{tabular}

\footnotetext{
${ }^{\mathrm{a}}$ The mean \pm standard deviation of the mean ( $\beta$-alanine: $\mathrm{n}=3$, other compounds: $\mathrm{n}=5$ ).

${ }^{\mathrm{b}}$ Not detected.
} 
Fig. 1

(a)

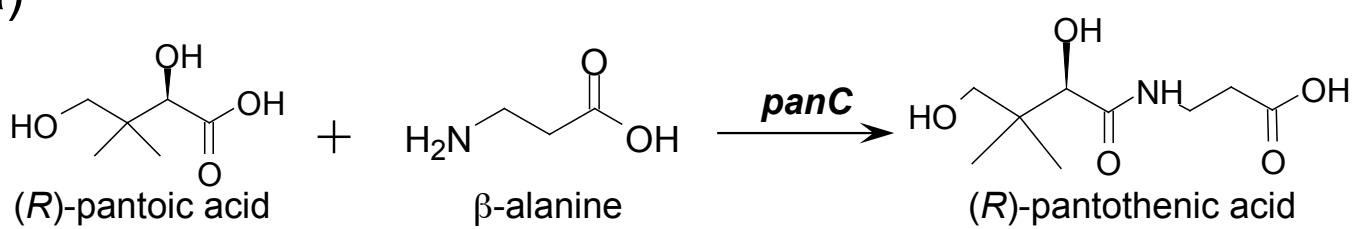

(b)

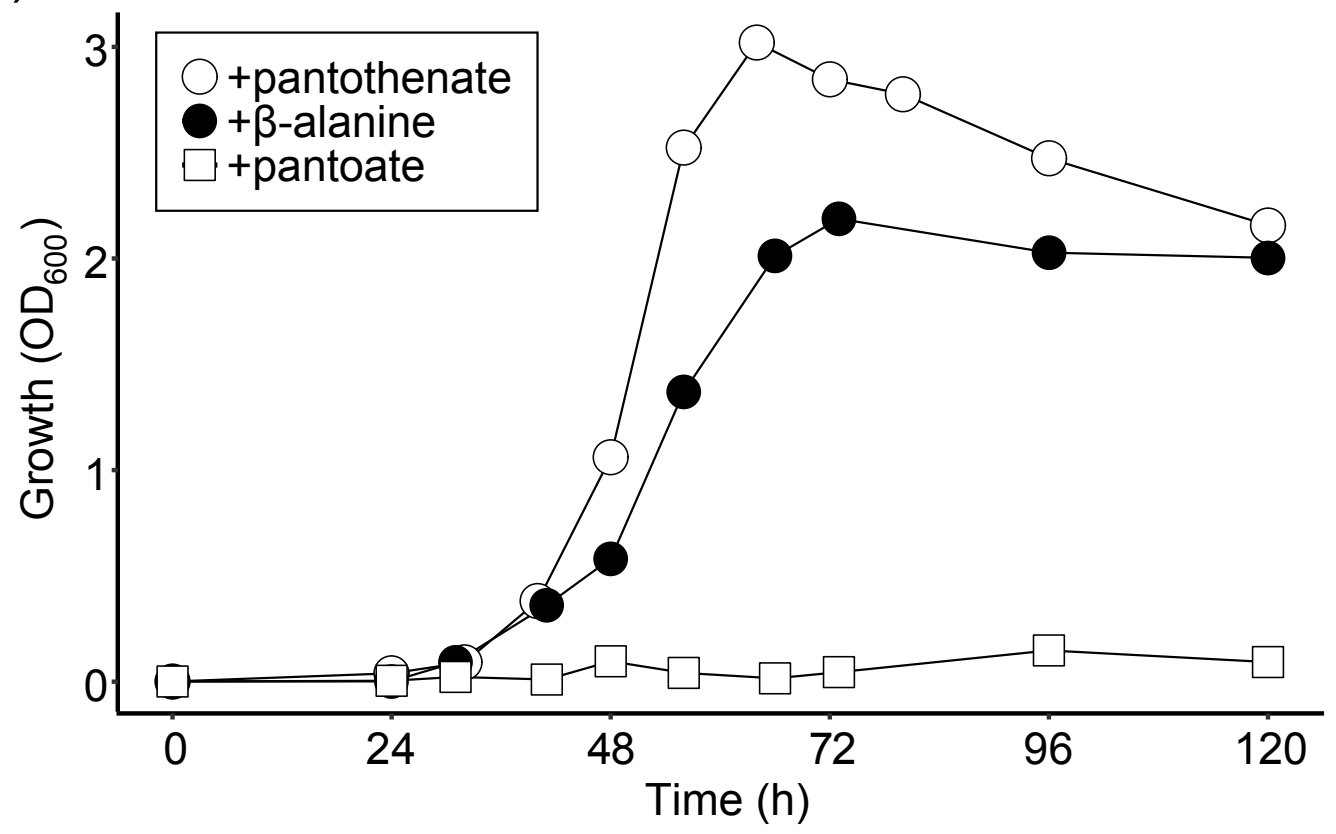


Fig. 2

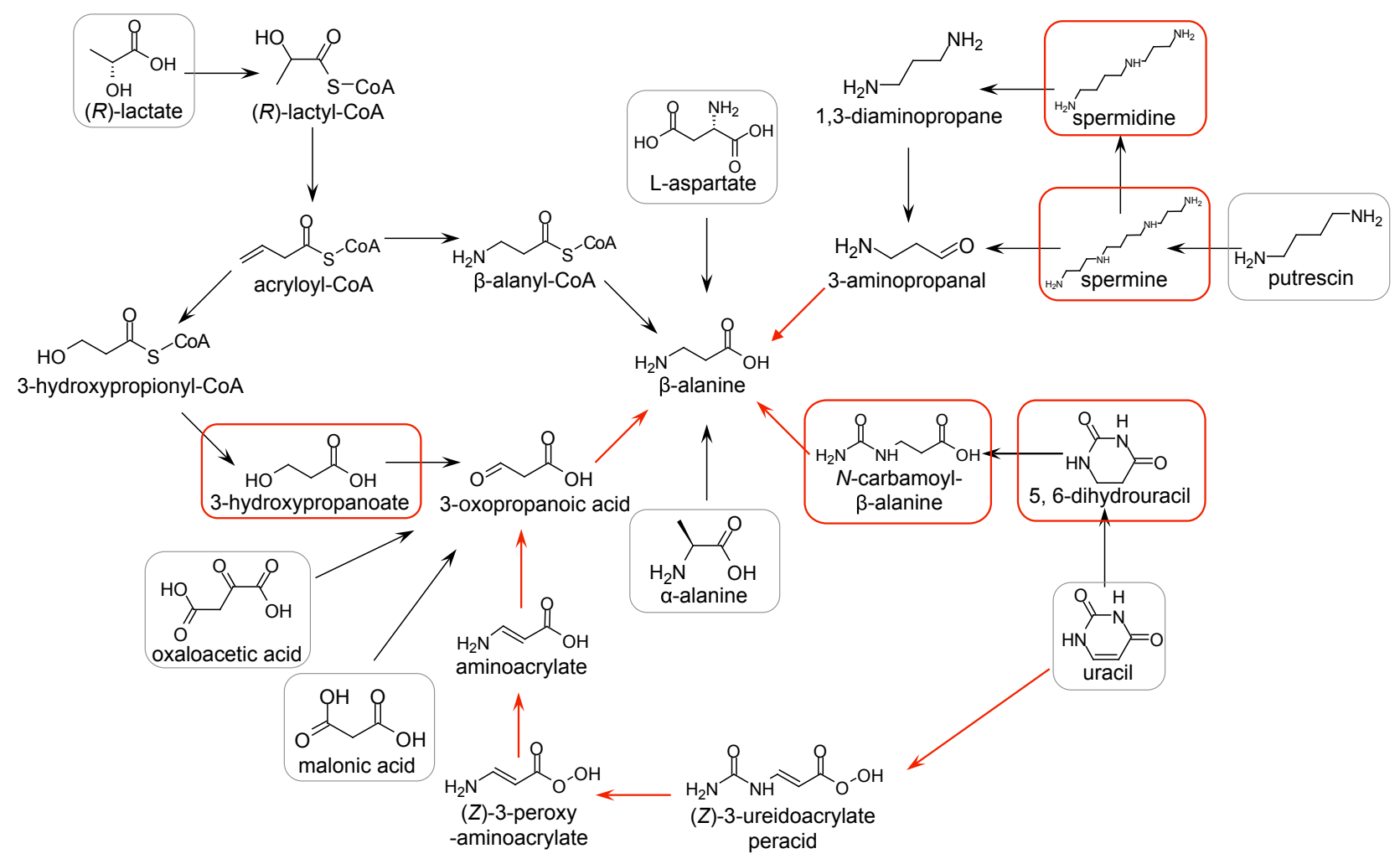


Fig. 3

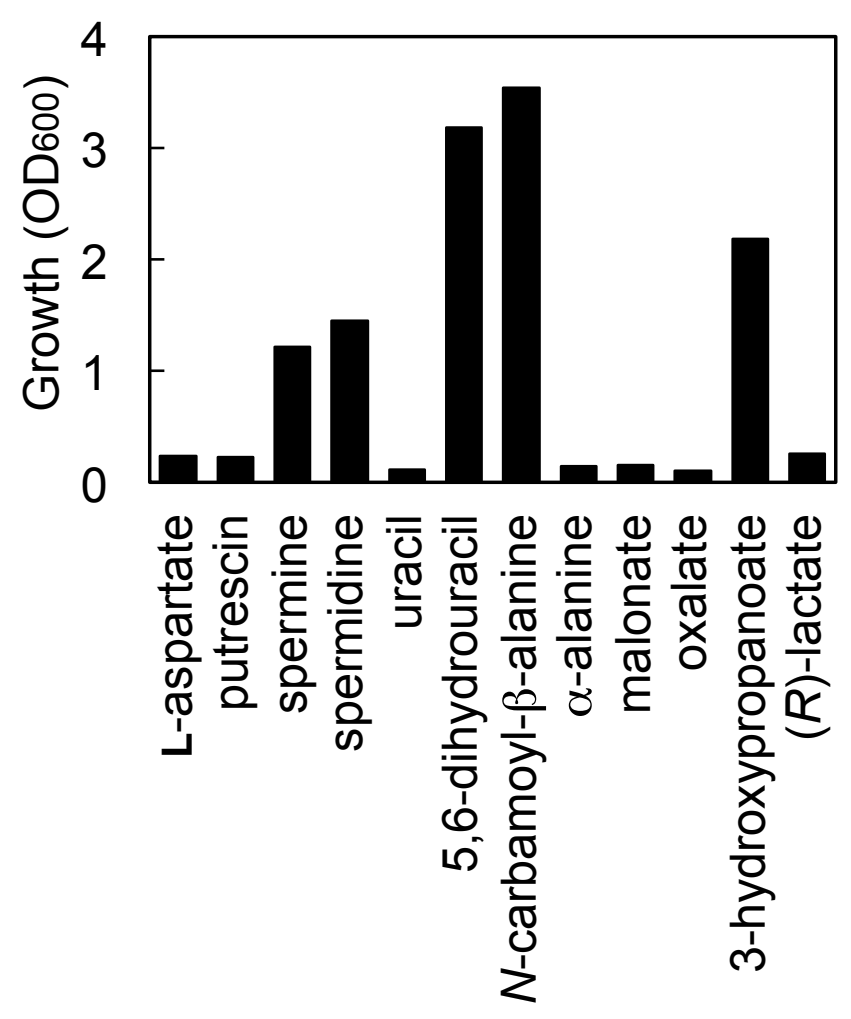


Fig. 4

(a)

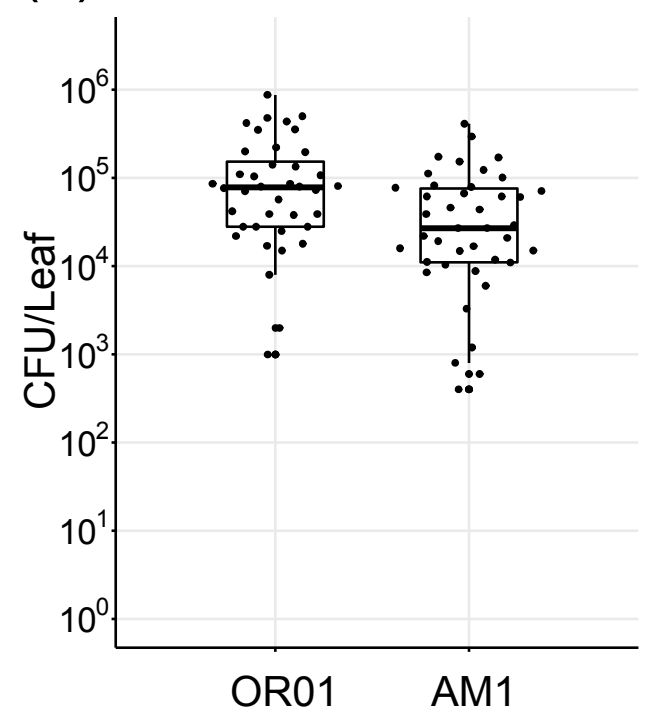

(b)

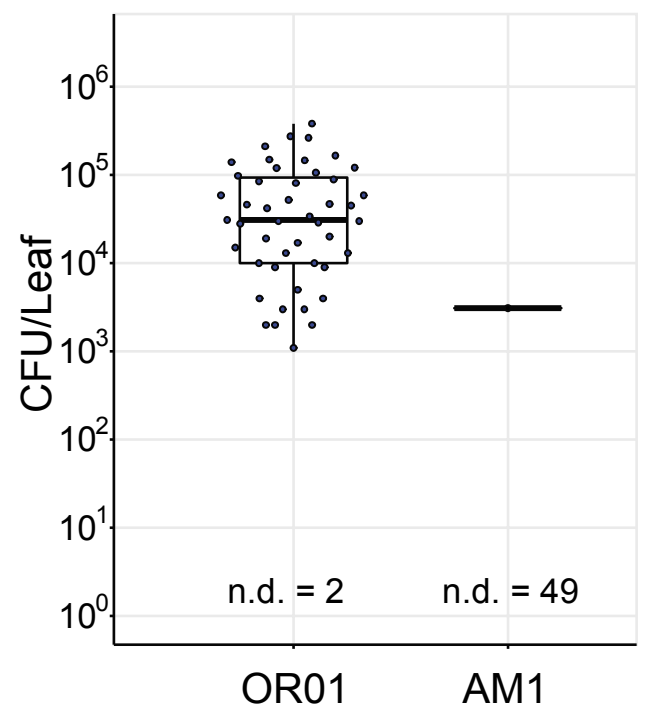


Fig. 5

(a)

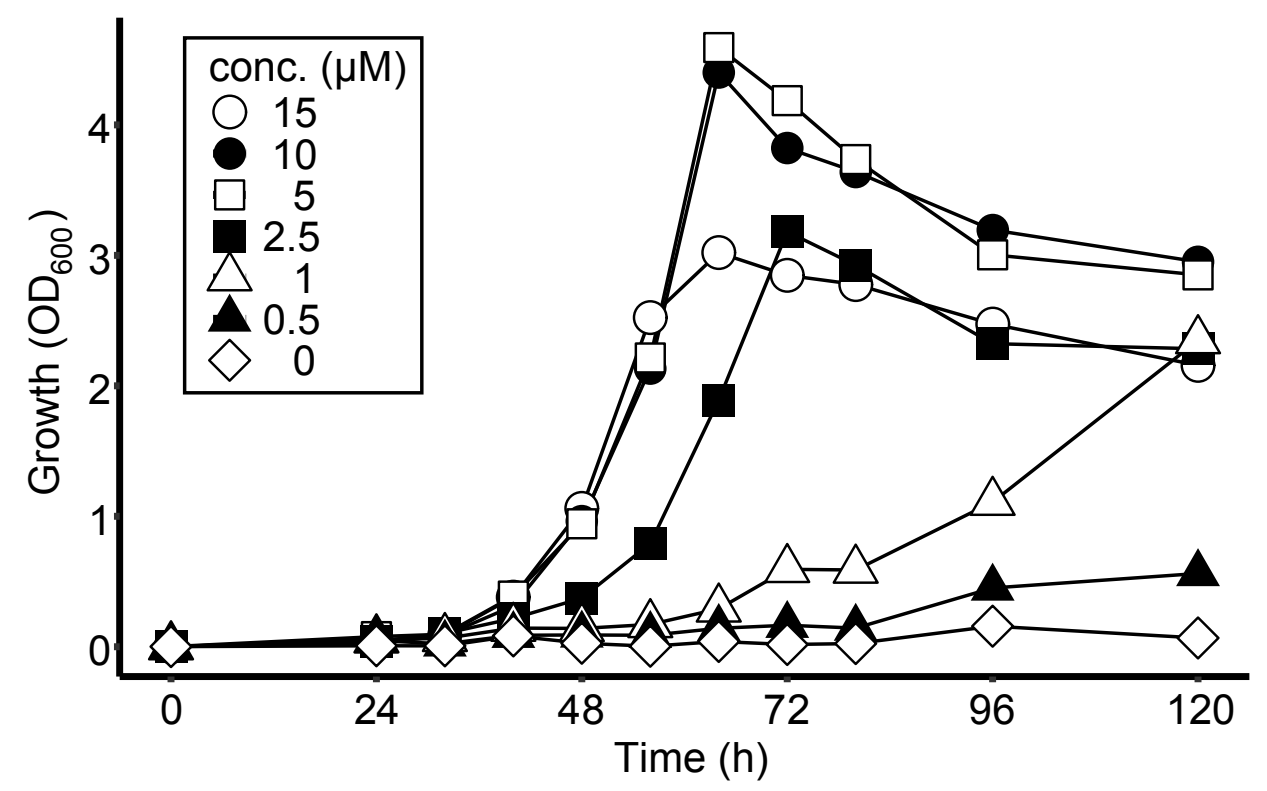

(b)

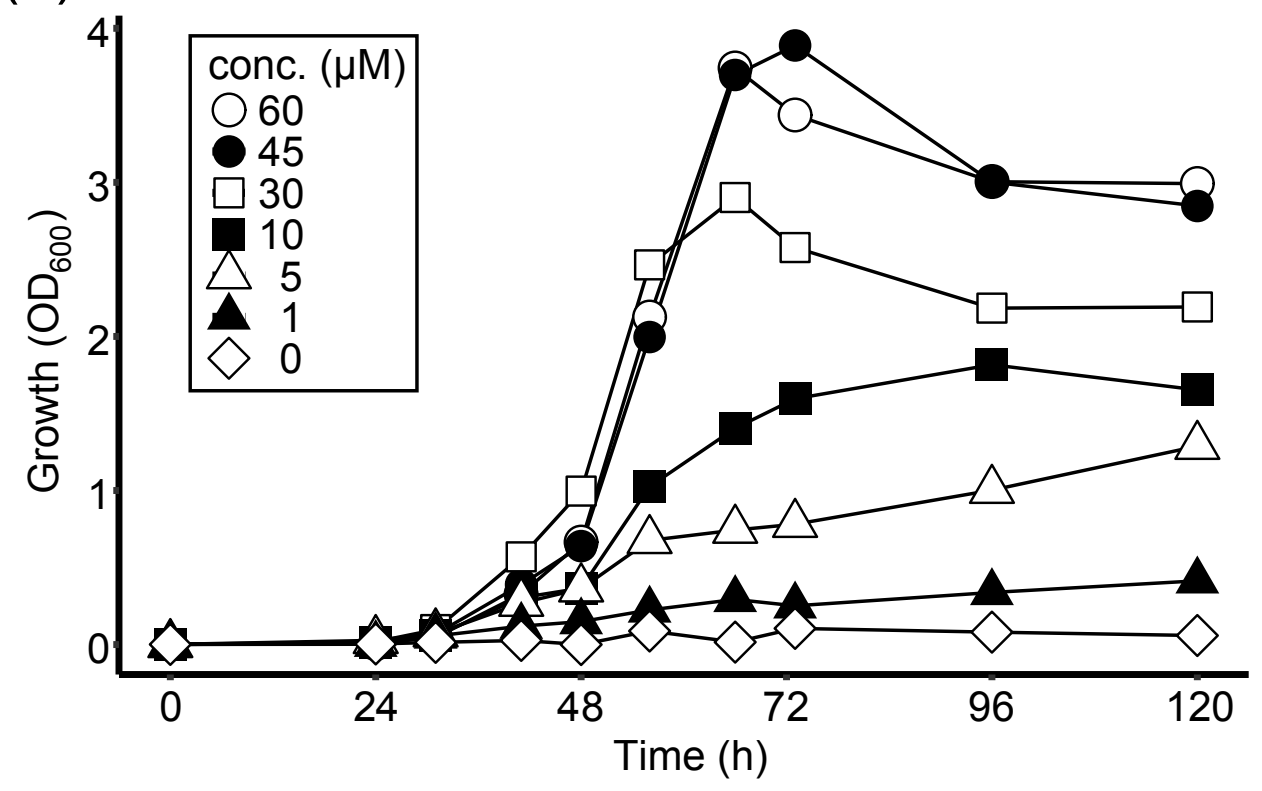


Table S1. Parameters for LC-MS/MS analysis.

\begin{tabular}{|c|c|c|c|c|c|c|c|c|c|c|}
\hline Compounds & $\mathrm{Q} 1(\mathrm{~m} / \mathrm{z})$ & Q3 (m/z) & $\mathrm{DP}(\mathrm{V})$ & $\mathrm{CE}(\mathrm{V})$ & CXP (V) & GS1 & GS2 & CUR & $\mathrm{TEM}\left({ }^{\circ} \mathrm{C}\right)$ & IS (V) \\
\hline pantothenate & 217.844 & 87.900 & & & & & & & & \\
\hline$N$-carbamoyl- $\beta$-alanine & 130.900 & 88.100 & -40 & -15 & -9 & 30 & 80 & 40 & 400 & -4500 \\
\hline 3-hydroxypropanoate & 88.900 & 58.900 & & & & & & & & \\
\hline spermine & 203.200 & 129.100 & & & & & & & & \\
\hline spermidine & 146.163 & 71.900 & 26 & 17 & 6 & 30 & 80 & 40 & 500 & 4500 \\
\hline 5,6-dihydrouracil & 115.200 & 69.900 & & & & & & & & \\
\hline$\beta$-alanine (derivatized) & 210.000 & 121.000 & 60 & 29 & 10 & 70 & 70 & 10 & 600 & 5500 \\
\hline
\end{tabular}

Q1, mass of precursor ion: Q3, mass of product ion: DP, declustering potential: CE, collision energy: CXP, collision exit potential:

GS1, ion source gas 1 (nebulizer gas): GS2, ion source gas 2 (heater gas): CUR, curtain gas: TEM, temperature: IS, ion spray voltage. 